\title{
Targeted Activation of Cholinergic Interneurons Accounts for the Modulation of Dopamine by Striatal Nicotinic Receptors
}

\author{
(D) Katherine R. Brimblecombe, ${ }^{1}$ (1) Sarah Threlfell, ${ }^{1,2}$ (DDaniel Dautan, ${ }^{3,4}$ Polina Kosillo, ${ }^{1, *}$ (D) Juan \\ Mena-Segovia, ${ }^{3,4}$ and ${ }^{-S t e p h a n i e ~ J . ~ C r a g g ~}{ }^{1,2}$
}

https://doi.org/10.1523/ENEURO.0397-17.2018

${ }^{1}$ Department of Physiology, Anatomy and Genetics, Sherrington Building, Oxford, UK, '2Oxford Parkinson's Disease Centre, University of Oxford, Oxford OX1 3PT, UK, ${ }^{3} \mathrm{MRC}$ Anatomical Neuropharmacology Unit, Department of

Pharmacology, Oxford, UK, and ${ }^{4}$ Center for Molecular and Behavioral Neuroscience, Rutgers University, Newark, NJ

\section{Visual Abstract}

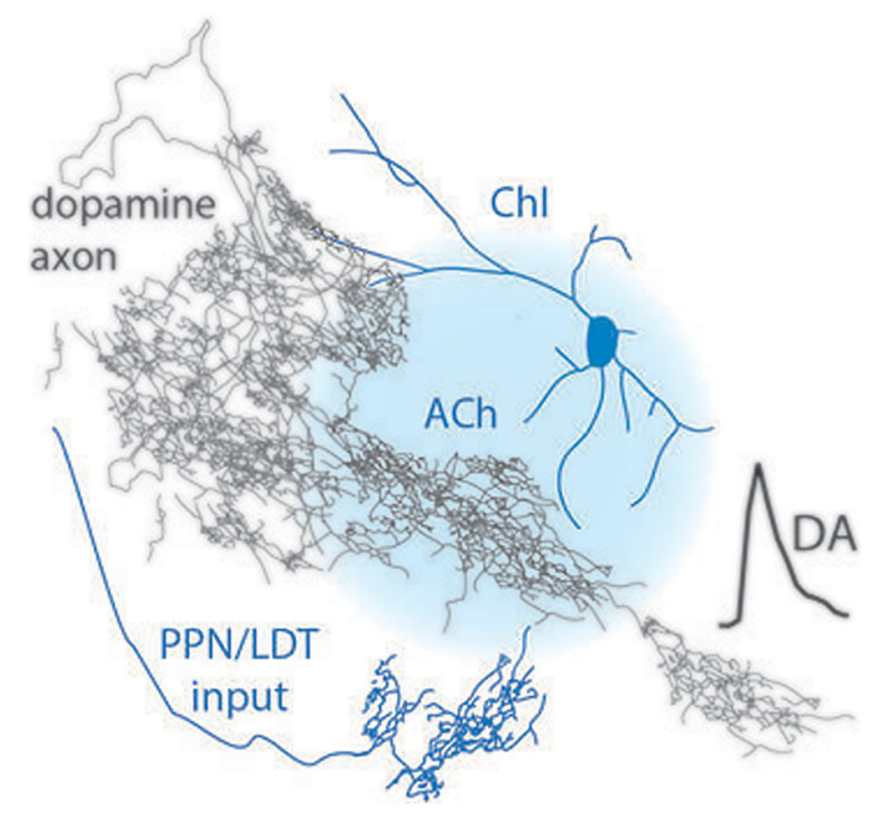

\section{Significance Statement}

Striatal acetylcholine (ACh) can powerfully regulate striatal dopamine (DA). Until recently, cholinergic interneurons were thought to be the only source of striatal $\mathrm{ACh}$, but brainstem cholinergic neurons have now been revealed to innervate striatum. Here, we use targeted optogenetic activation in rats to explore which input accounts for the rapid regulation of striatal DA release. We find that targeted activation of striatal cholinergic interneurons in rat striatal slices can reproduce how striatal ACh drives and modulates dopamine release in mouse, but we could not find comparable evidence for a role for brainstem cholinergic inputs. Activation of cholinergic interneurons alone can reproduce the previously documented effects of activating all striatal ACh systems on DA release. 
Striatal dopamine (DA) is a major player in action selection and reinforcement. DA release is under strong local control by striatal ACh acting at axonal nicotinic ACh receptors (nAChRs) on DA axons. Striatal nAChRs have been shown to control how DA is released in response to ascending activity from DA neurons, and they also directly drive DA release following synchronized activity in a small local cholinergic network. The source of striatal ACh has been thought to arise solely from intrinsic cholinergic interneurons (Chls), but recent findings have identified a source of cholinergic inputs to striatum from brainstem nuclei, the pedunculopontine nucleus (PPN) and laterodorsal tegmentum (LDT). Here, we used targeted optogenetic activation alongside DA detection with fast-scan cyclic voltammetry to test whether Chls alone and/or brainstem afferents to the striatum can account for how ACh drives and modulates DA release in rat striatum. We demonstrate that targeted transient light activation of rat striatal Chls drives striatal DA release, corroborating and extending previous observations in mouse to rat. However, the same light stimulation targeted to cholinergic brainstem afferents did not drive DA release, and nor did it modulate DA release activated subsequently by electrical stimulation, whereas targeted activation of Chls did so. We were unable to obtain any evidence for DA modulation by PPN/LDT stimulation. By contrast, we could readily identify that striatal Chls alone are sufficient to provide a source of ACh that powerfully regulates DA via nAChRs.

Key words: Acetylcholine; brainstem; cholinergic interneurons; dopamine; nicotinic receptors; striatum

\section{Introduction}

The striatum is the primary input-receiving nucleus of the basal ganglia, and the modulation of information flow through the striatum is crucial to basal ganglia function including motor control and reward-driven behaviors. Two key striatal neuromodulators are dopamine (DA) and acetylcholine (ACh). Striatal ACh plays powerful roles not only in regulating excitability of striatal neurons, but also in directly regulating DA transmission (Zhou et al., 2001; Rice and Cragg, 2004; Zhang and Sulzer, 2004; Cragg, 2006; Threlfell et al., 2010, 2012; Cachope et al., 2012). Activation of $\beta 2$-containing $\mathrm{nAChRs}$ on dopaminergic axons (Jones et al., 2001) promotes DA release probability, but in turn subsequently leads to short-term depression of further release, making DA release insensitive to depolarization frequency (Rice and Cragg, 2004; Zhang and Sulzer, 2004; Wang et al., 2014). It has therefore been proposed that in vivo, when striatal cholinergic interneurons (Chls) pause their firing in response to salient and conditioned stimuli (Aosaki et al., 1994; Apicella et al., 1997), or when nicotine causes receptor desensitization, nAChRs will turn off and allow DA release to better reflect the frequency of activity in DA neurons (Rice and Cragg, 2004; Cragg, 2006). In addition, synchronized activation of a small network of striatal cholinergic inputs can directly drive DA release via activation of striatal nAChRs,

Received November 18, 2017; accepted September 16, 2018; First published October 12, 2018

The authors declare no competing financial interests.

Author Contributions: All authors designed research; K.R.B., P.K., S.T., and

D.D. performed research; and K.R.B., J.M.-S., and S.J.C. wrote the paper.

Funded by MRC (grant MR/K013866/1) and Parkinson's UK (J-1403; G-1305).

*P. Kosillo's current address is Dept. of Molecular and Cell Biology, Berkeley, University of California, CA.

Address correspondence to Stephanie J. Cragg, Department of Physiology, Anatomy and Genetics, University of Oxford, OX1 3PT, UK. E-mail: stephanie.cragg@dpag.ox.ac.uk.

https://doi.org/10.1523/ENEURO.0397-17.2018

Copyright $\odot 2018$ Brimblecombe et al.

This is an open-access article distributed under the terms of the Creative Commons Attribution 4.0 International license, which permits unrestricted use, distribution and reproduction in any medium provided that the original work is properly attributed. bypassing activation of DA neurons in the midbrain (Cachope et al., 2012; Threlfell et al., 2012).

The source of striatal ACh has long been thought to be exclusively striatal Chls, which constitute only $\sim 1 \%-2 \%$ of all striatal neurons (Kawaguchi et al., 1995; Rymar et al., 2004). Despite being few in number, Chls have an extensive influence throughout the striatum through their extended dendritic and axonal arbors (Hoover et al., 1978; Graveland and DiFiglia, 1985; Kawaguchi, 1993; Descarries and Mechawar, 2000). However, recent work has revealed an additional source of ACh inputs to striatum in rats that arise from the pedunculopontine nucleus (PPN) and laterodorsal tegmental nucleus (LDT) of the brainstem. These inputs project respectively to $\mathrm{CPu}$ and NAc (plus medial $\mathrm{CPu}$ ), express the vesicular $\mathrm{ACh}$ transporter (vAChT), and give rise to striatal synapses (Dautan et al., $2014,2018)$. The identification of this brainstem input to striatum has led to the question of whether these inputs provide a source of ACh that might mediate the previously documented effects of ACh on striatal DA.

Previous studies of DA regulation using strategies that target optogenetic constructs to striatal Chls (Cachope et al., 2012; Threlfell et al., 2012) have primarily used an AAV serotype (AAV5) that does not select for anterograde over retrograde expression, and thus intrinsic striatal Chls as well as any ACh afferent inputs will have expressed channelrhodopsin2 (ChR2) in those studies. Therefore, we explored here the roles of cholinergic brainstem afferents versus Chls as sources of the striatal ACh that drives and modulates striatal DA release (Zhou et al., 2001; Rice and Cragg, 2004; Exley et al., 2008, 2012; Zhang et al., 2009; Threlfell et al., 2010, 2012; Cachope et al., 2012), by targeting expression of ChR2 to striatal Chls or to cholinergic brainstem neurons. We used rats as a species of choice because brainstem cholinergic input has been best described in this species (Dautan et al., 2014) and also, to validate the role of ACh in DA regulation across rodent species.

\section{Methods}

\section{Stereotaxic surgery}

Adult $(250 \mathrm{~g}-350 \mathrm{~g})$ male Long Evans ChAT::cre ${ }^{+}$rats were maintained on a 12-h light/dark cycle (light on at 
7:00 AM) and had ad libitum access to water and food. All procedures were performed in accordance with the Society of Neuroscience policy on the use of animals in neuroscience and the Animals (Scientific Procedures) Act, 1986 (UK), under the authority of a Project License approved by the Home Office and the local ethics review committee.

All surgical procedures were performed during deep isoflurane anesthesia ( $2 \%$ in $\mathrm{O}_{2}$, IsoFlo, Schering-Plow). ChAT:: $\mathrm{cre}^{+}$rats were injected in each hemisphere with adeno-associated virus (AAV) of serotype 2 for anterograde-specific expression (Salegio et al., 2013; Dautan et al., 2016), carrying the fusion genes for the enhanced yellow fluorescent protein (eYFP) and channelrhodopsin (ChR2; AAV2-EF1a-DIO-ChR2-eYFP, Gene Therapy Center Virus Vector Core, University of North Carolina). The viral vectors were injected in the right brainstem (3 injections of $500 \mathrm{nl}$ each in the LDT: AP $-8.5, \mathrm{ML}+1.0$, DV -6.0 , the caudal part of the PPN: AP $-7.8, \mathrm{ML}+1.8$, DV -6.8 , and the rostral part of the PPN: AP $-7.3, \mathrm{ML}+1.8$, DV 7.2) and in the left striatum to target Chls (4 injections of $500 \mathrm{nl}$ each in the ventral DLS: AP $+0.5, \mathrm{ML}+3.0$, DV -5.0; dorsal DLS: AP +0.5, ML +2.0, DV -4.0; ventral DMS: AP + 0.5, ML +2.0, DV -5.0; dorsal DMS: AP + 0.5, $\mathrm{ML}+3.0$, DV -4.0). All injections were made using a $1-\mu l$ syringe (Neuros 7001, Hamilton) at a rate of $50 \mathrm{nl} / \mathrm{min}$ and left to diffuse for $5 \mathrm{~min}$ before retraction of the syringe. Animals were monitored during recovery. LDT/PPN injections result in ChR2-eYFP expression in $66 \% \pm 7 \%$ of LDT/PPN cholinergic neurons, with $91.4 \% \pm 0.5 \%$ of ChR2-eYFP neurons being immunopositive for ChAT. Light activation of these inputs can successfully drive a cholinergic output from terminals in VTA (Dautan et al., 2016).

\section{Slice preparation}

Six to eight weeks after AAV2 injections, rats were killed by decapitation under isoflurane-induced anesthesia, and brains were rapidly removed; $300-\mu \mathrm{m}$ coronal striatal sections were taken in ice-cold buffer containing, in mM: 120 $\mathrm{NaCl}, 20 \mathrm{NaHCO}_{3}, 6.7 \mathrm{HEPES}$ acid, $5 \mathrm{KCl}, 3.3 \mathrm{HEPES}$ salt, $2 \mathrm{CaCl}_{2}, 2 \mathrm{MgSO}_{4}, 1.2 \mathrm{KH}_{2} \mathrm{PO}_{4}$, and 10 glucose, saturated with $95 \% \mathrm{O}_{2} / 5 \% \mathrm{CO}_{2}$. Slices were kept at room temperature in HEPES-based buffer for at least $1 \mathrm{~h}$.

\section{Fast-scan cyclic voltammetry (FCV)}

DA release was monitored in acute slices using FCV. Slices were superfused in a recording chamber with bicarbonate-buffered artificial cerebrospinal fluid (aCSF) containing, in mM: $124.3 \mathrm{NaCl}, 26 \mathrm{NaHCO}_{3}, 3.8 \mathrm{KCl}, 2.4$ $\mathrm{CaCl}_{2}, 1.3 \mathrm{MgSO}_{4}, 1.23 \mathrm{KH}_{2} \mathrm{PO}_{4}$, and 10 glucose, saturated with $95 \% \mathrm{O}_{2} / 5 \% \mathrm{CO}_{2}$ at $31-33^{\circ} \mathrm{C}$. Evoked extracellular DA concentration ([DA $]_{\circ}$ ) was monitored using FCV at 7-10- $\mu \mathrm{m}$-diameter carbon-fiber microelectrodes (CFM) fabricated in-house (tip length 50-100 $\mu \mathrm{m}$ ) and a Millar voltammeter (Julian Millar, Barts and the London School of Medicine and Dentistry). In brief, a triangular voltage wave form (range -700 to $+1300 \mathrm{mV}$ versus $\mathrm{Ag} / \mathrm{AgCl}$ ) was applied at $800 \mathrm{~V} / \mathrm{s}$ at a scan frequency of $8 \mathrm{~Hz}$. Electrodes were switched out of circuit between scans. Electrodes were calibrated using 1-2 $\mu \mathrm{M}$ DA in each experimental medium. Calibration solutions were prepared immediately before calibration from a $2.5-\mathrm{mm}$ stock solution in $0.1 \mathrm{M}$ $\mathrm{HClO}_{4}$ stored at $4^{\circ} \mathrm{C}$. Signals were attributable to DA by the potentials for peak oxidation and reduction currents (oxidation peak: $+500-600 \mathrm{mV}$, reduction peak: $\sim-200$ $\mathrm{mV}$ ).

\section{Electrical stimulation}

DA release was evoked by a local bipolar concentric $\mathrm{Pt} / \mathrm{Ir}$ electrode $(25-\mu \mathrm{m}$ diameter; $\mathrm{FHC})$ placed $\sim 100 \mu \mathrm{m}$ from the CFM. Stimulus pulses (200- $\mu$ s duration) were given at $0.6 \mathrm{~mA}$ (perimaximal in control conditions). Electrical stimulations were repeated at 2.5-min intervals, which allow stable release to be sustained over several hours. Each stimulus type was repeated in triplicate in a random order. When directly comparing DA release evoked by light versus electrical stimuli, stimuli at $25 \mathrm{~Hz}$ were used to allow for ChR2 reactivation. When exploring changes in frequency sensitivity of DA release, electrical stimulations of single pulses (1p) and 4 pulses (4p) at 100 $\mathrm{Hz}$ were used because the ratio of DA released by $4 \mathrm{p} /$ $100 \mathrm{~Hz}$ versus $1 p(4 p: 1 p)$ is very sensitive to $n A C h R$ activity (Rice and Cragg, 2004). When nAChRs are active, $4 p: 1 p$ is $\sim 1$, indicating a large degree of short-term depression, whereas when nAChRs are blocked or desensitized, the $4 \mathrm{p}: 1 \mathrm{p}$ can be $\sim 4$.

\section{Optical stimulation}

Light stimulation of ChR2-expressing Chls and brainstem afferents in striatum was via a 470-nm LED (OptoLED, Cairn Research), which illuminated the full field of view (2.2-mm diameter, 10× water-immersion objective). TTLdriven light pulses (2-ms duration, $\sim 6.5 \mathrm{~mW}$, Thor labs optical power meter) were applied singly or in trains $(4-10$ pulses, $10-25 \mathrm{~Hz}$ ). In some experiments, light activation of Chls that was subthreshold for evoking DA release was desired, for comparison with brainstem activation. This was achieved by stimulating and recording in areas of sparse Chl transfection, i.e., posterior and lateral CPu. Electrical and optical stimulations at a given site were alternated.

\section{Drugs}

Dihydro- $\beta$-erythroidine $(\mathrm{DH} \beta \mathrm{E})$ was purchased from Ascent Scientific. All other reagents were purchased from Sigma Aldrich. Stock solutions were made to $1000 \times$ final concentrations in $\mathrm{H}_{2} \mathrm{O}$ and stored at $-20^{\circ} \mathrm{C}$. Drugs were diluted to their required concentrations in aCSF immediately before use.

\section{Immunocytochemistry and site marking}

Images of ChR2-eYFP expression were captured after fixation. Slices were fixed (PFA 4\% formaldehyde) for at least $2 \mathrm{~d}$, washed in PBS, and resectioned to $40 \mu \mathrm{m}$. Sections were mounted on gelled slides with Vectashield (Vector labs), and ChR2-eYFP fluorescence was imaged using an Olympus BX41 microscope with Q-Click cooled monochrome CCD camera (Olympus Medical).

ChR2-eYFP fluorescence in Chls was readily visible in $300 \mu \mathrm{m}$ acute living brain slices in the recording chamber under water-immersion optics using (nonpulsing) activa- 
Table 1. Statistical analysis

\begin{tabular}{|c|c|c|c|}
\hline Location & Data structure & Type of test & Power \\
\hline a & Normal & 2-way ANOVA with Sidak posttest & $\begin{array}{l}\text { Interaction: } F_{1,11}=8.5, p=0.014 \\
\text { post hoc test: } 1 p \text { vs } 4 p \mathrm{DH} \beta \mathrm{E}, p<0.05\end{array}$ \\
\hline b & Normal & 2-way ANOVA & $\begin{array}{l}\text { Interaction: } F_{1,6}=17.1, p=0.006 \\
\text { post hoc test: } 1 p \text { vs } 4 p, p<0.05\end{array}$ \\
\hline c & Normal & 2-way ANOVA (repeated measures) & $\begin{array}{l}\text { Effect of light: } F_{1,4}=0.19, p=0.68 ; \text { pre-flash } \times \\
\text { pulse number interaction, } F_{1,4}=0.02, p=0.88\end{array}$ \\
\hline d & Normal & 2-way ANOVA & $\begin{array}{l}\text { Effect of region: } F_{1,18}=8.7, p=0.004 \text {; posttest, } \\
\text { with vs without prepulse: Chls, } p<0.01\end{array}$ \\
\hline e & Normal & 2-way ANOVA & Effect of light: $F_{1,12}=0.2, p=0.67$ \\
\hline
\end{tabular}

tion of eYFP using OptoLED (505 nm), whereas ChR2eYFP-expressing fibers from PPN/LDT were not. We therefore marked striatal recording sites with FluoSpheres, to determine post hoc whether they were within an area of ChR2-expressing cholinergic brainstem innervation. Recording sites were located in central to medial striatum consistent with the organization of cholinergic inputs to striatum from PPN/LDT and were labeled by replacing the CFM with a micropipette and injecting 0.5$1.0 \mu \mathrm{l}$ of $1-\mu \mathrm{m}$-diameter red FluoSpheres (Invitrogen) after recordings. Slices were fixed (PFA 4\% formaldehyde) for at least $2 \mathrm{~d}$, washed in PBS, and resectioned to $40 \mu \mathrm{m}$. Sections were mounted on gelled slides with Vectashield (Vector labs) and imaged using an Olympus BX41 microscope with Q-Click cooled monochrome CCD camera (Olympus Medical). Monochrome images of striatal ChR2eYFP expression and FluoSpheres were each captured and pseudo-colored using Q-capture Pro7 and optimized using histogram equalization. Only recordings that were confirmed post hoc to be in striatal areas within a region of ChR2-eYFP-expressing neuropil (see example in Fig. $2 A)$ were included for analysis.

To confirm that striatal neurons transduced by AAV2 injected in rat striatum were cholinergic, striatal sections were incubated in a blocking solution consisting of $10 \%$ normal donkey serum (NDS) in PBS containing 1\% Triton $\mathrm{X}-100$ for $1 \mathrm{~h}$ minimum. Sections were incubated with previously validated antibodies against GFP (1:1000, raised in rabbit; Invitrogen, A21311) and an antibody against ChAT (raised in goat; 1:500 dilution in 1\% NDS, $0.03 \%$ Triton X-100 in PBS; Millipore, AB144P) followed by several washes in PBS and incubation in an Alexa Fluor 488-conjugated donkey anti-rabbit antibody (1:1000 dilution, Jackson Immunoresearch) or CY5-conjugated donkey anti-goat antibody (1:1000, Jackson Immunoresearch).

\section{Statistics}

Data are expressed as mean \pm SEM. Data at each site were averaged from at least three repeat recordings for each stimulus, and where appropriate was normalized to $1 p$ release in control conditions. Population means were compared using two-way ANOVA with repeated measures and Sidak's multiple comparison $t$ test as appropriate. Superscript letters listed with $p$-values correspond to the statistical tests shown in Table 1.

\section{Results}

\section{Striatal ACh from rat Chls drives dopamine release}

To obtain striatal expression of ChR2-eYFP in either Chls or afferents from PPN/LDT, we injected anterogradespecific AAV2-packaged constructs into either the striatum or PPN/LDT, respectively, of ChAT-Cre rats. Striatal injections resulted in detectable ChR2-eYFP expression in striatum and an absence of retrograde transduction of ChR2-eYFP expression in PPN/LDT cholinergic neurons in brainstem, whereas PPN/LDT injections resulted in ChR2-eYFP expression in PPN/LDT and in neuropil in striatum (Fig. 1A), as shown previously following striatal versus PPN/LDT injections of AAV2-EF1a-DIO-YFP in ChAT-Cre rats (Dautan et al., 2014). ChR2-eYFP expression was confirmed as originating from neurons immunopositive for ChAT in striatum (Fig. 1B), as has been confirmed previously for expression in PPN/LDT neurons following brainstem injections (Dautan et al., 2016).

We first sought to test whether targeted activation of striatal Chls is able to directly drive DA release. To confirm recording site viability, we initially explored DA release in response to electrical stimuli (single pulses, $1 \mathrm{p}$, or trains of 4 pulses, $4 p$ at $25 \mathrm{~Hz}$ ); evoked striatal DA release in rat did not vary with stimulation protocol in drug-free control conditions (Fig. 1C,D), consistent with previous observations in mice and guinea pig (Rice and Cragg, 2004; Zhang et al., 2009; Exley et al., 2012). DA release was strongly regulated by $n A C h R s:$ when $n A C h R s$ were inhibited by antagonist $\mathrm{DH} \beta \mathrm{E}(1 \mu \mathrm{M})$, peak [DA] evoked by $1 \mathrm{p}$ was reduced and by $4 \mathrm{p}(25 \mathrm{~Hz})$ was slightly facilitated (Fig. $1 C, D$ ), consistent with previous findings (Rice and Cragg, 2004; Zhang and Sulzer, 2004; Zhang et al., 2009). Thus, inhibition of nAChRs promoted the sensitivity of DA release to axonal activity (Fig. 1D; 2-way ANOVA drug $\times$ pulse number interaction $F_{1,11}=8.5, p=0.014^{\text {a }}$; post hoc test: $1 p$ versus $4 p \mathrm{DH} \beta E, p<0.05)$. In the same recording sites, in the absence of $\mathrm{DH} \beta \mathrm{E}$, targeted activation of ChR2-expressing Chls using blue light pulses (1p or $4 p$, $25 \mathrm{~Hz}$ ) also evoked DA release and was insensitive to pulse number (Fig. 1E-G), as shown previously in mice using AAV5-packaged ChR2 (Threlfell et al., 2012). Lightactivated DA release was abolished in the presence of $\mathrm{DH} \beta \mathrm{E}(1 \mu \mathrm{M})$, showing the dependence on nAChRs. Since Chls but not brainstem neurons express ChR2 in these animals (Fig. 1A), these data show that that targeted activation of Chls alone can reproduce in rats the previously documented effects in mice of activating all striatal 

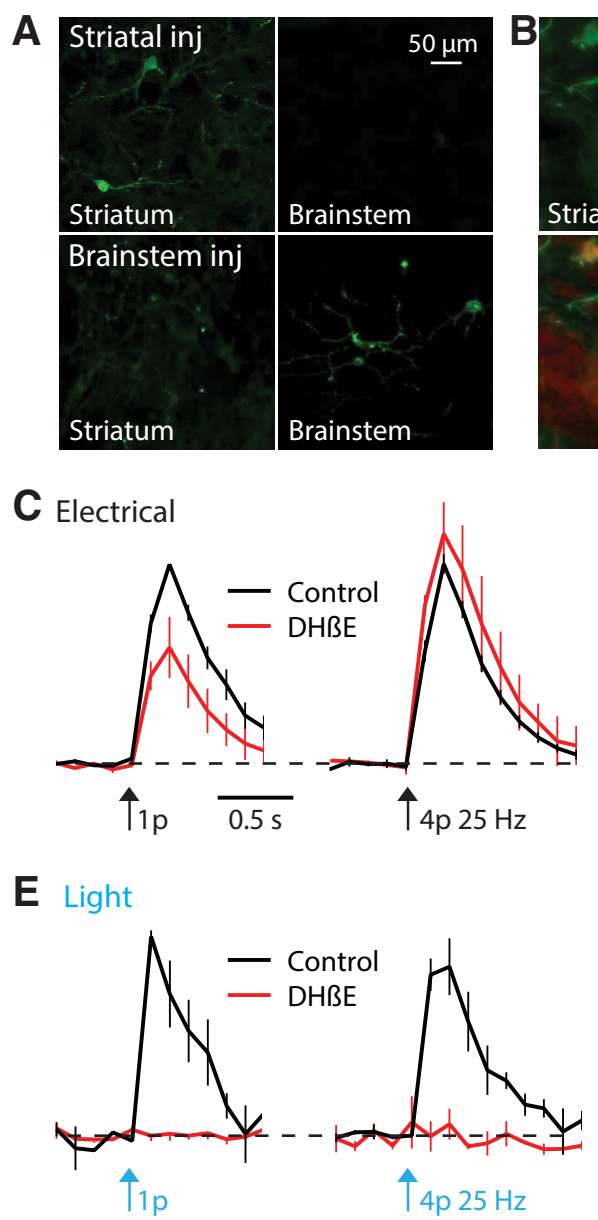
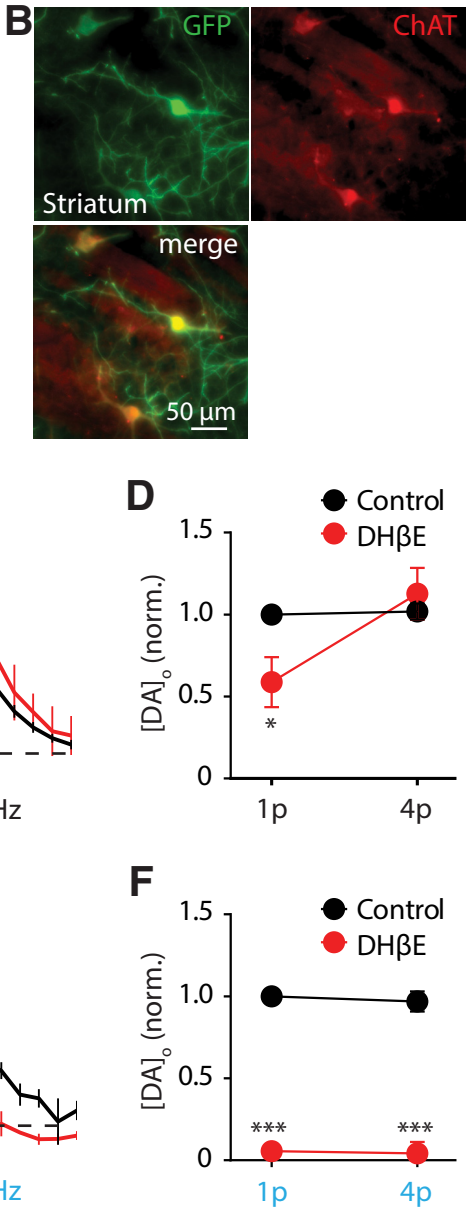

Figure 1. Light activation of rat striatal cholinergic interneurons evokes striatal dopamine release. $\boldsymbol{A}$, ChR2-eYFP detected in striatum (left) and PPN/LDT (right) after AAV2 injection to PPN/LDT (lower), and detected in striatum only after injection to striatum (upper), fixed tissue. $\boldsymbol{B}$, GFP-immunopositive soma after striatal injection were co-immunopositive for ChAT. C, $\boldsymbol{E}$, Mean profiles of [DA] \pm SEM versus time evoked by 1 or 4 pulses $(25 \mathrm{~Hz}$ ) of electrical $(C)$ or light $(E)$ stimulation, in control drug-free conditions or in the presence of $\mathrm{DH} \beta \mathrm{E}(1 \mu \mathrm{M})$. Data are normalized to $1 \mathrm{p}$ peak $[\mathrm{DA}]_{\mathrm{o}}$ in control conditions. Control conditions (black lines), $\mathrm{DH} \beta \mathrm{E}(\mathrm{red}$ lines). $\boldsymbol{D}, \boldsymbol{F}$, Mean peak $[D A]_{0}$ normalized to control 1p for electrical $(\boldsymbol{D})$ and light-evoked DA release $(\boldsymbol{F})$ in control (black) and in the presence of $\mathrm{DH} \beta \mathrm{E}$ (red). $n=3$ rats, $n=4$ sites. Two-way ANOVA with Sidak posttest comparisons of control versus $\mathrm{DH} \beta \mathrm{E}: * p<0.05, * * * p<$ 0.001 .

ACh systems on driving DA release (Fig. $1 E, F ; n=4$ slices from 3 rats). Evoked [DA] ranged from 0.2 to $1.2 \mu \mathrm{M}$ for single electrical pulses and from 0.13 to $1.7 \mu \mathrm{M}$ for single light pulses.

\section{Striatal ACh from brainstem afferents does not drive DA release}

To explore whether striatal ACh afferents from PPN/ LDT can drive DA release in response to these brief stimulations, when activation of either Chls alone (see Fig. 1) or all striatal ACh networks strongly drive $D A$ release (Cachope et al., 2012; Threlfell et al., 2012), we injected an AAV2 containing ChR2-eYFP constructs into the right PPN/LDT of ChAT-cre rats (Fig. 2A). This protocol leads to ChR2-eYFP expression in $\sim 70 \%$ of PPN cholinergic neurons (Dautan et al., 2016). In contrast to the high density of ChR2-eYFP-expressing striatal fibers seen after striatal injections, striatal ChR2-eYFP expression after PPN/LDT injections was not visible by fluorescence imaging during recordings in live slices. Expression of ChR2 was corrob- orated only after recording, following injection of red FluoSpheres to mark recording sites using a published protocol (Brimblecombe and Cragg, 2015), followed by fixation, to confirm whether recordings were in an area of innervation by ChR2-expressing brainstem afferents (Fig. 2A).

In striatal sites with confirmed ChR2-eYFP-expressing brainstem fibers, brief light pulses did not evoke detectable levels of $[D A]_{\circ}$ even with trains of 10 pulses $(10 \mathrm{~Hz}$; Fig. 2B,C). By contrast, electrical stimulation at the same recording sites reliably evoked DA release, proving site viability (Fig. $2 B, C$ ). Furthermore, light activation of the opposite hemisphere where Chls were transfected with ChR2-eYFP also reliably evoked DA release, even with single pulses (Fig. 2B,C). To test if activation of cholinergic brainstem afferents was driving DA release below our DA detection threshold, we applied an inhibitor of DA uptake, cocaine $(5 \mu \mathrm{M})$, to enhance any evoked DA transients. In the presence of cocaine, [DA] evoked by electrical stimuli (single pulses) were large and prolonged, but by contrast, 
A
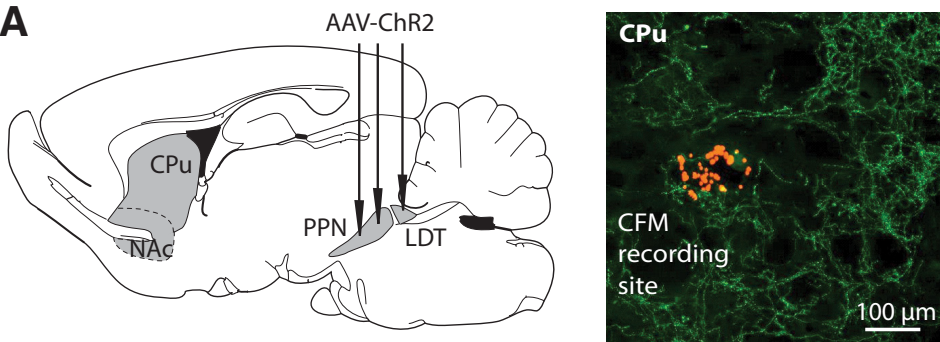

B
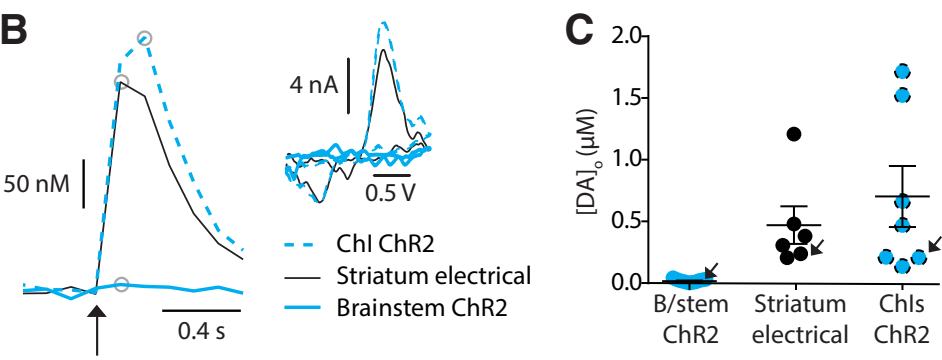

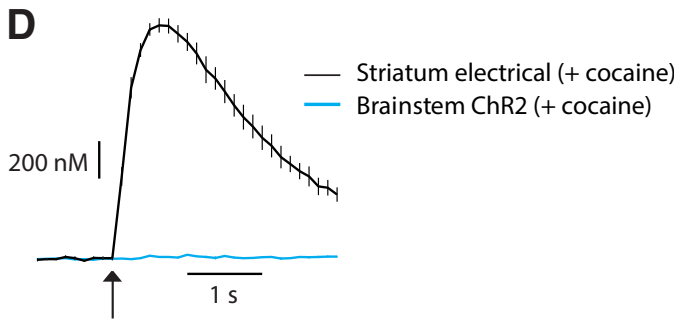

Figure 2. Light activation of striatal cholinergic brainstem afferents with brief stimuli does not reveal dopamine release. $\boldsymbol{A}$, Left, cartoon indicating PPN/LDT injections sites, and right, recording site labeled with red FluoSpheres in an area of cholinergic brainstem innervation indicated by ChR2-eYFP fluorescence. $\boldsymbol{B}$, Example profiles of $[\mathrm{DA}]_{\mathrm{o}}(\mu \mathrm{M})$ versus time evoked by either light activation of Chls (Chl ChR2, blue dashed, 1p), electrical stimulation of striatum (black solid line, 1p), or light activation of brainstem cholinergic afferents (brainstem ChR2, blue solid line, 10p $10 \mathrm{~Hz}$ ). Inset, corresponding voltammograms for DA following each activation type for site indicated in $\boldsymbol{A}$, at time point indicated on profiles by gray circles. $\boldsymbol{C}$, Peak evoked [DA] $(\mu \mathrm{M})$ for each recording site and stimulation methods, with mean \pm SEM indicated. Arrows indicate data points and sites shown in $\boldsymbol{B}$. $\boldsymbol{D}$, Mean profiles of current detected at DA oxidation potential $( \pm$ SEM) versus time evoked by striatal electrical stimulation (1p; black) or light stimulation of ChR2-expressing cholinergic brainstem afferents (10p $10 \mathrm{~Hz}$; blue) in the presence of cocaine (5 $\mu \mathrm{m} ; n=10$ observations from 3 sites).

light activation of brainstem afferents did not evoke detectable [DA] (Fig. 2D). These observations suggest that activation of cholinergic brainstem afferents alone does not generate a sufficient ACh source with these protocols in slices, to explain how striatal DA release is locally driven by striatal ACh.

\section{Brainstem ACh afferents do not modulate electrically evoked DA release}

We tested the possibility that brainstem afferents might nonetheless be able to modulate presynaptic excitability of DA axons but in a manner that is below the threshold for directly driving DA release. We tested whether we could expose a role for brainstem ACh in the modulation of DA release evoked by other stimuli. We established a stimulus protocol in which DA release was driven by local electrical stimulation with or without a prior light pulse train in ChR2-expressing striatum. We hypothesized that if prior light stimulation caused $\mathrm{ACh}$ release to reach nAChRs on DA axons, then it might modulate nAChR activity and/or promote desensitization, and consequently change the frequency sensitivity of electrically evoked DA release that is critically determined by $\mathrm{nAChRs}$ (Cragg, 2006). We used a light pulse train $(10 \mathrm{p} / 10 \mathrm{~Hz})$ that commenced $0.5 \mathrm{~s}$ before electrical stimulation to detect any effects of light stimulation alone, as well as subsequent effects on combined electrical stimulation.

We first established the effects of combined light and electrical stimulation on striatal DA when ChR2 was expressed by Chls. We selected striatal regions where the density of ChR2-eYFP-expressing fibers from Chls was sparse, to be more comparable to the innervation by brainstem afferents. In regions sparsely innervated by ChR2-expressing Chls, electrical stimuli alone readily evoked DA release, but trains of light pulses $(10 \mathrm{p} / 10 \mathrm{~Hz})$ did not drive $[D A]$ to a detectable level (see Fig. $3 A$ ). A prestimulation of Chls with light pulses that commenced $0.5 \mathrm{~s}$ before an electrical stimulus did not evoke detectable $[D A]_{\circ}$ but nonetheless modified $[D A]_{\circ}$ evoked by electrical stimulation: there was a reduction in $[D A]$ 。 evoked by a single electrical pulse and a tendency toward a facilitation of $[D A]_{\circ}$ evoked by a burst of electrical pulses $(4 \mathrm{p} / 100 \mathrm{~Hz}$; compared to electrical stimulus alone; Fig. $3 A, B$; 2-way repeated measures ANOVA: light condition 

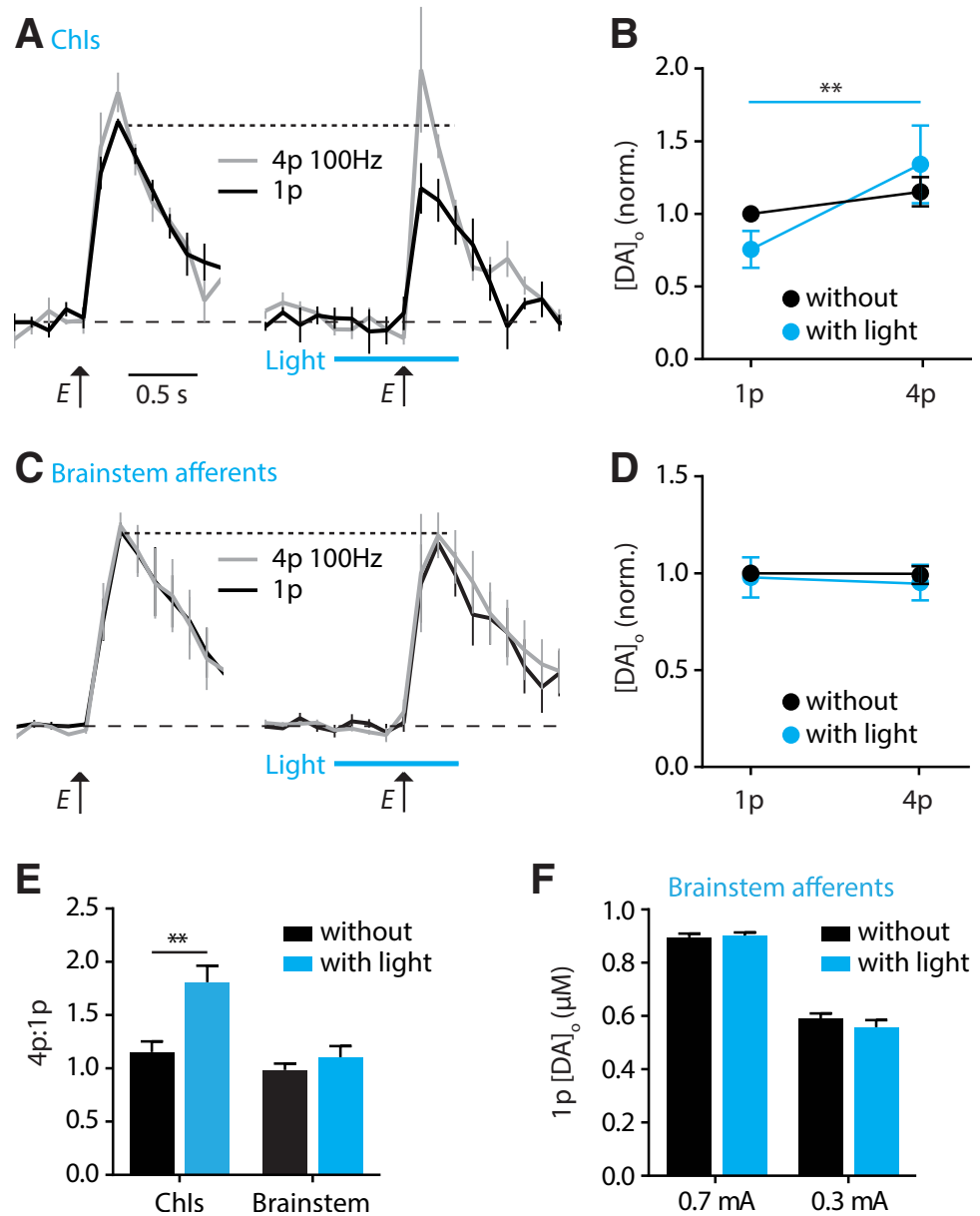

Figure 3. Light activation of striatal cholinergic interneurons but not brainstem afferents readily modulates dopamine release. $\boldsymbol{A}$, $\boldsymbol{C}$, Mean profiles of $[D A]_{0} \pm S E M$ versus time evoked by 1 or 4 pulses $(100 \mathrm{~Hz})$ of electrical (E) stimulation alone (left), or with stimulation with light $(10 \mathrm{p} / 10 \mathrm{~Hz}$ beginning $0.5 \mathrm{~s}$ before electrical; right) to stimulate ChR2 expressed in either Chls $(\boldsymbol{A})$ or brainstem afferents $(\boldsymbol{C})$. Data are normalized to $1 \mathrm{p}$ control (without light). $\boldsymbol{B}, \boldsymbol{D}$, Summary data showing mean peak [DA] \pm SEM evoked by the electrical stimulation normalized to control 1p, for ChR2-expressing Chls $(\boldsymbol{B})$ or brainstem afferents $(\boldsymbol{D})$ with and without light activation (blue versus black). Two-way ANOVA with repeated measures with Sidak posttest comparisons of $1 p$ versus $4 p: * * p<0.01 ; N . S$., $p>0.05$. $\boldsymbol{E}$, Ratio of $[\mathrm{DA}]_{\text {。 }}$ evoked by 4 versus 1 electrical pulses $(100 \mathrm{~Hz})$ with and without light stimulation (blue versus black) of either Chls or striatal brainstem afferents. Light pre-stimulation of Chls but not brainstem afferents significantly potentiated $4 \mathrm{p}: 1 \mathrm{p}$ ratio of electrically evoked DA release (2-way ANOVA; Sidak posttest for with versus without light: Chl: $t_{18}=4.1, * * p<0.01$; brainstem: $t_{18}$ $=0.58, p>0.05)$. $\boldsymbol{F}$, Mean peak $1 \mathrm{p}[\mathrm{DA}]_{\mathrm{o}} \pm \mathrm{SEM}$, evoked by $0.7 \mathrm{~mA}$ and $0.3 \mathrm{~mA}$ electrical stimuli, with and without light prestimulation of brainstem afferents. Two-way ANOVA; Sidak posttest with versus without light: $0.7 \mathrm{~mA}: \mathrm{t}_{12}=0.29, p>0.05 ; 0.3 \mathrm{~mA}: t_{12}=1.2$, $p>0.05$. Data are in $\mu \mathrm{m}, n=4$ rats, $n=6$ sites.

$\times$ pulse number interaction: $\left.F_{1,6}=17.1, p=0.006^{\mathrm{b}}\right)$. There was a resulting increase in the ratio of $[D A]_{\circ}$ evoked electrically by $4 p$ versus $1 p$ (Fig. $3 A, B$; post hoc test, $1 p$ versus $4 p$ with light: $p<0.05$ ). This modulation of evoked $[D A]_{\circ}$ is consistent with reduced activation of nAChRs (Rice and Cragg, 2004; Zhang and Sulzer, 2004) at the time of electrical stimulation, confirming that light activation of Chls that was subthreshold for directly driving DA release was nonetheless able to modulate nAChRs and DA release.

We then tested the effect of prior light stimulation of cholinergic brainstem afferents on subsequent electrically evoked DA release. Light activation of brainstem afferents in striatum did not drive detectable [DA $]_{\circ}$ (as in Fig. 2), and furthermore, did not modulate $[D A]_{\text {。 }}$ evoked by electrical stimulation given $0.5 \mathrm{~s}$ later (Fig. $3 C, D$; 2-way repeated measures ANOVA, effect of light: $F_{1,4}=0.19, p=0.68$; pre-flash $\times$ pulse number interaction, $F_{1,4}=0.02, p=$ $\left.0.88^{\mathrm{C}}\right)$. The ratio of [DA] evoked electrically by $4 p$ versus $1 p$ was not enhanced by light prestimulation of brainstem afferents (Fig. 3E, 2-way ANOVA, effect of region: $F_{1,18}=$ $8.7, p=0.004$; posttest, with versus without pre-pulse: Chls, $p<0.01^{\mathrm{d}}$ ).

Finally, in the event that subtle effects of prior ACh action at $n A C h R s$ were masked by the stronger subsequent electrical stimuli, we tested whether we could expose an effect of a light prestimulation for a lower electrical stimulation current. A lower electrical current evoked $[D A]_{0}$ that were $\sim$ two-thirds of those previously, but nonetheless, prestimulation of ChR2-expressing brainstem afferents with light pulse trains did not modify [DA] further (Fig. 3F, 2-way ANOVA: effect of light: $F_{1,12}=0.2, p=0.67^{\mathrm{e}}$ ). 


\section{Discussion}

We show here, using specific targeting of ChR2 to either striatal Chls or brainstem cholinergic neurons of PPN/LDT, that the powerful effects of striatal ACh and $\mathrm{nAChRs}$ on DA release reported previously ex vivo can be reproduced by targeted activation of Chls. Striatal Chls and $n A C h R s$ can directly drive DA release, and also modulate DA release driven by activity generated in DA neurons, shown here in rats, as reported previously in mice using nonspecific ACh activation (Rice and Cragg, 2004; Zhang and Sulzer, 2004; Cachope et al., 2012; Threlfell et al., 2012). By contrast, while PPN/LDT nuclei can modulate somatodendritic DA neuron activity (Dautan et al., 2016), we could not find any evidence that similar stimulation protocols could recruit PPN/LDT afferents in striatum to regulate DA release from axons.

Two cholinergic sources for regulation of striatal DA

$\mathrm{ACh}$ is well known to modulate DA transmission through two major cholinergic pathways that have previously been assumed to be distinct. First, roles of the cholinergic brainstem for modulating DA neuron activity and consequent DA release have previously been established. Cholinergic neurons of the brainstem make synaptic contacts with midbrain DA neurons (Beninato and Spencer, 1988; Bolam et al., 1991), and direct activation of midbrain ACh receptors or PPN/LDT cholinergic axons modulates the discharge properties of DA neurons and, consequently, the downstream release of DA in the striatum (Blaha and Winn, 1993; Dautan et al., 2016). These studies indicate that cholinergic PPN/LDT neurons are able to modulate DA release via inputs to midbrain DA neurons. Second, a role for striatal acetylcholine systems, nAChRs, and nicotine in modulating DA axonal release directly has become well documented, and striatal ACh has been shown to bypass midbrain DA neurons and directly drive DA release (Rice and Cragg, 2004; Zhang and Sulzer, 2004; Cragg, 2006; Cachope et al., 2012; Threlfell et al., 2012). The neurons responsible for local striatal modulation were assumed to be only the intrinsic interneurons, the Chls. However, the identification of cholinergic afferents in striatum arising from the PPN/LDT (Dautan et al., 2014) suggested that brainstem inputs might be partly responsible for local striatal modulation of DA by contributing as an additional source of striatal ACh. However, we were unable to obtain any evidence here for either ACh release or DA modulation by presynaptic nAChRs following PPN/LDT stimulation.

\section{Cholinergic interneurons but not brainstem afferents account for rapid regulation of striatal DA by nAChRs}

Our experiments explored whether protocols that have been used previously to activate striatal ACh systems, and that drive and powerfully modulate striatal DA via nAChRs, primarily involve Chls or brainstem afferents. Our data indicate that whereas ACh from Chls could account for previously documented effects of nAChRs on striatal DA, we could not show the same was true for brainstem afferents. Single light flashes in Chls are suffi- cient to drive DA release directly, but by contrast, single flashes or even more sustained stimulation of brainstem afferents did not allow us to detect any DA. We were unable to find any further signs of modulation of nAChRs on DA axons in a protocol designed to explore whether activation of brainstem afferents is capable of modulating DA release evoked by other stimuli. As a positive control, we showed that pre-activation of sparsely labeled Chls, that in isolation was unable to drive DA release, was nonetheless able to modulate electrically evoked DA release, and significantly increase the $4 p: 1 p$ ratio. This increase in $4 \mathrm{p}: 1 \mathrm{p}$ ratio is consistent with a decrease in activation of $n A C h R s$ on DA axons at the point of electrical stimulation. This decrease in $\mathrm{nAChR}$ activity could result from initial $A C h$ release leading to a depression in ACh re-release by the subsequent electrical pulse, or to some $\mathrm{nAChR}$ desensitization, to which $\mathrm{nAChRs}$ are particularly susceptible (Zhou et al., 2001; Quick and Lester, 2002; Rice and Cragg, 2004). By contrast, prestimulation of brainstem afferents with light had no measurable effect on subsequent electrically evoked $[D A]_{0}$ or on $4 \mathrm{p}: 1 \mathrm{p}$ ratio.

These data suggest that any ACh released by activation of brainstem afferents alone is unable to reach sufficient levels to modulate $\mathrm{nAChR}$ s on DA axons. The more readily detectable role for Chls in gating striatal DA, and also for cortical or thalamic glutamate inputs to Chls (Kosillo et al., 2016) than for brainstem inputs, is in keeping with the relative visibility of striatal YFP expression noted in $300-\mu \mathrm{m}$-thick slices under the recording microscope. This conclusion is also consistent with the finding that deletion of brainstem cholinergic neurons does not affect electrically evoked dopamine release in striatal slices (Patel et al., 2012). We cannot exclude the possibility that ACh might not be released from PPN/LDT terminals in striatum in ex vivo slices. This caveat does not detract from the principal conclusion that striatal Chls are entirely sufficient to account for current observations of the modulation of DA by striatal ACh. Our findings do not necessarily preclude brainstem afferents from locally regulating DA release during other conditions, e.g., in vivo, when they might modulate the dynamic activity in vivo of networks of striatal neurons or Chls (Dautan et al., 2018), which might in turn subsequently regulate DA release.

\section{Summary and conclusions}

In summary, we show that Chls readily drive DA release in rat, as shown previously in mouse, and that striatal modulation of DA transmission by $\mathrm{nAChRs}$ and nicotine can readily be reproduced by targeted activation of Chls but not brainstem afferents. Brainstem afferents were not sufficient to drive or modulate striatal DA release under the experimental paradigms used here as previously, in which Chl function dominates.

\section{References}

Aosaki T, Tsubokawa H, Ishida A, Watanabe K, Graybiel AM, Kimura M (1994) Responses of tonically active neurons in the primate's striatum undergo systematic changes during behavioral sensorimotor conditioning. J Neurosci 14:3969-3984. CrossRef

Apicella P, Legallet E, Trouche E (1997) Responses of tonically discharging neurons in the monkey striatum to primary rewards 
delivered during different behavioral states. Exp Brain Res 116: 456-466. CrossRef

Beninato M, Spencer RF (1988) The cholinergic innervation of the rat substantia nigra: a light and electron microscopic immunohistochemical study. Exp Brain Res 72:178-184. CrossRef

Blaha CD, Winn P (1993) Modulation of dopamine efflux in the striatum following cholinergic stimulation of the substantia nigra in intact and pedunculopontine tegmental nucleus-lesioned rats. $\mathrm{J}$ Neurosci 13:1035-1044. CrossRef

Bolam JP, Francis CM, Henderson Z (1991) Cholinergic input to dopaminergic neurons in the substantia nigra: a double immunocytochemical study. Neuroscience 41:483-494. CrossRef

Brimblecombe KR, Cragg SJ (2015) Substance P weights striatal dopamine transmission differently within the striosome-matrix axis. J Neurosci 35:9017-9023. CrossRef

Cachope R, Mateo Y, Mathur BN, Irving J, Wang HL, Morales M, Lovinger DM, Cheer JF (2012) Selective activation of cholinergic interneurons enhances accumbal phasic dopamine release: setting the tone for reward processing. Cell Rep 2:33-41. CrossRef

Cragg SJ (2006) Meaningful silences: how dopamine listens to the ACh pause. Trends Neurosci 29:125-131. CrossRef

Dautan D, Huerta-Ocampo I, Valencia M, Kondabolu K, Gerdjikov TV, Mena-Segovia J (2018) Cholinergic midbrain afferents modulate striatal circuits and shape encoding of action control. bioRxiv August 8, 2018. CrossRef

Dautan D, Huerta-Ocampo I, Witten IB, Deisseroth K, Bolam JP, Gerdjikov T, Mena-Segovia J (2014) A major external source of cholinergic innervation of the striatum and nucleus accumbens originates in the brainstem. J Neurosci 34:4509-4518. CrossRef

Dautan D, Souza AS, Huerta-Ocampo I, Valencia M, Assous M, Witten IB, Deisseroth K, Tepper JM, Bolam JP, Gerdjikov TV, Mena-Segovia J (2016) Segregated cholinergic transmission modulates dopamine neurons integrated in distinct functional circuits. Nat Neurosci 19:1025-1033. CrossRef

Descarries L, Mechawar N (2000) Ultrastructural evidence for diffuse transmission by monoamine and acetylcholine neurons of the central nervous system. Prog Brain Res 125:27-47.

Exley R, Clements MA, Hartung H, Mclntosh JM, Cragg SJ (2008) $\alpha 6$-containing nicotinic receptors dominate the nicotine control of dopamine neurotransmission in nucleus accumbens. Neuropsychopharmacol 33:2158-2166. CrossRef Medline

Exley R, Mclntosh JM, Marks MJ, Maskos U, Cragg SJ (2012) Striatal alpha5 nicotinic receptor subunit regulates dopamine transmission in dorsal striatum. J Neurosci 32:2352-2356. CrossRef

Graveland GA, DiFiglia M (1985) The frequency and distribution of medium-sized neurons with indented nuclei in the primate and rodent neostriatum. Brain Res 327:307-311. Medline

Hoover DB, Muth EA, Jacobowitz DM (1978) A mapping of the distribution of acetycholine, choline acetyltransferase and acetylcholinesterase in discrete areas of rat brain. Brain Res 153:295306. Medline
Jones IW, Bolam JP, Wonnacott S (2001) Presynaptic localisation of the nicotinic acetylcholine receptor beta2 subunit immunoreactivity in rat nigrostriatal dopaminergic neurones. J Comp Neur 439: 235-247. CrossRef

Kawaguchi Y (1993) Physiological, morphological, and histochemical characterization of three classes of interneurons in rat neostriatum. J Neurosci 13:4908-4923. CrossRef

Kawaguchi Y, Wilson CJ, Augood SJ, Emson PC (1995) Striatal interneurones: chemical, physiological and morphological characterization. Trends Neurosci 18:527-535. CrossRef

Kosillo P, Zhang YF, Threlfell S, Cragg SJ (2016) Cortical control of striatal dopamine transmission via striatal cholinergic interneurons. Cereb Cortex 11:4160-4169. CrossRef

Patel JC, Rossignol E, Rice ME, Machold RP (2012) Opposing regulation of dopaminergic activity and exploratory motor behavior by forebrain and brainstem cholinergic circuits. Nat Commun 3:1172. CrossRef

Quick MW, Lester RA (2002) Desensitization of neuronal nicotinic receptors. J Neurobiol 53:457-478. CrossRef Medline

Rice ME, Cragg SJ (2004) Nicotine amplifies reward-related dopamine signals in striatum. Nat Neurosci 7:583-584. CrossRef

Rymar VV, Sasseville R, Luk KC, Sadikot AF (2004) Neurogenesis and stereological morphometry of calretinin-immunoreactive GABAergic interneurons of the neostriatum. J Comp Neur 469: 325-339. CrossRef

Salegio EA, Samaranch L, Kells AP, Mittermeyer G, San Sebastian W, Zhou S, Beyer J, Forsayeth J, Bankiewicz KS (2013) Axonal transport of adeno-associated viral vectors is serotypedependent. Gene Ther 20:348-352. CrossRef

Threlfell S, Clements MA, Khodai T, Pienaar IS, Exley R, Wess J, Cragg SJ (2010) Striatal muscarinic receptors promote activity dependence of dopamine transmission via distinct receptor subtypes on cholinergic interneurons in ventral versus dorsal striatum. J Neurosci 30:3398-3408. CrossRef

Threlfell S, Lalic T, Platt NJ, Jennings KA, Deisseroth K, Cragg SJ (2012) Striatal dopamine release is triggered by synchronized activity in cholinergic interneurons. Neuron 75:58-64. CrossRef

Wang L, Shang S, Kang X, Teng S, Zhu F, Liu B, Wu Q, Li M, Liu W, Xu H, Zhou L, Jiao R, Dou H, Zuo P, Zhang X, Zheng L, Wang S, Wang C, Zhou Z (2014) Modulation of dopamine release in the striatum by physiologically relevant levels of nicotine. Nat Commun 5:3925. CrossRef

Zhang H, Sulzer D (2004) Frequency-dependent modulation of dopamine release by nicotine. Nat Neurosci 7:581-582. CrossRef

Zhang L, Doyon WM, Clark JJ, Phillips PE, Dani JA (2009) Controls of tonic and phasic dopamine transmission in the dorsal and ventral striatum. Mol Pharmacol 76:396-404. CrossRef

Zhou FM, Liang Y, Dani JA (2001) Endogenous nicotinic cholinergic activity regulates dopamine release in the striatum. Nat Neurosci 4:1224-1229. CrossRef 